\title{
Effect of Using Personal Protective Equipment during the COVID- 19 Pandemic on the Quality Indicators of Screening Colonoscopies
}

\author{
Subin Chirayath $\left(\mathbb{D},{ }^{1}\right.$ Janak Bahirwani $\mathbb{D}^{1},{ }^{1}$ Parampreet Kaur $\mathbb{D}^{2},{ }^{2}$ Noel Martins $\left(\mathbb{D},{ }^{3}\right.$ \\ and Ronak Modi $(\mathbb{D})^{3}$ \\ ${ }^{1}$ Department of Internal Medicine, St. Luke's University Hospital, USA \\ ${ }^{2}$ Department of Data Management and Outcomes Assessment, St. Luke's University Hospital, USA \\ ${ }^{3}$ Department of Gastroenterology, St. Luke's University Hospital, USA
}

Correspondence should be addressed to Subin Chirayath; subin.chirayath@sluhn.org

Received 3 May 2021; Revised 11 June 2021; Accepted 2 July 2021; Published 31 July 2021

Academic Editor: Paraskevas Gkolfakis

Copyright ( 92021 Subin Chirayath et al. This is an open access article distributed under the Creative Commons Attribution License, which permits unrestricted use, distribution, and reproduction in any medium, provided the original work is properly cited.

\begin{abstract}
Background. Coronavirus Disease 2019 (COVID-19) has affected many facets of the practice of medicine including screening colonoscopies. Aims. Our study looks to observe if there has been an effect on the quality of colonoscopies, as indicated by quality measures such as the cecal intubation rate (CIR), cecal intubation time (CIT), scope withdrawal time (SWT), and adenoma detection rate (ADR) with the adoption of standard COVID-19 precautions. Methods. We conducted a retrospective chart review to analyze the effects of the COVID-19 pandemic on screening colonoscopies. The study utilized data on CIR, CIT, SWT, and ADR from outpatient, nonemergent procedures conducted at 3 endoscopy suites of St. Luke's University Health Network. All inpatient and emergent procedures were excluded. Results. Our study demonstrated that the total number of screening colonoscopies was decreased between 2019 and 2020 (318 in 2019 vs. 157 in 2020, $p=0.005$ ). CIT (320 \pm 105 seconds in 2019 vs. $392 \pm 107$ seconds in 2020, $p=0.001)$ and SWT $(706 \pm 232$ seconds in 2019 vs. $830 \pm 241$ seconds in 2020, $p=0.001)$ were increased while CIR $(98.2 \%$ in 2019 vs. $96.6 \%$ in $2020, p=0.04)$ was decreased between 2019 and 2020 likely due to PPE introduction. ADR was similar between the two groups (38.23 (12.50-66.66) in 2019 vs. $38.18(16.66-66.00)$ in 2020, $p=0.8)$. Conclusion. Our study showed that quality indices for screening colonoscopies like CIR, CIT, and SWT were negatively impacted during the COVID-19 time period. ADR, however, was similar. Thus, the efficiency of the procedures was affected by the use of PPE but it did not affect the colonoscopy's clinical benefit.
\end{abstract}

\section{Introduction}

SARS-CoV-2, causing Coronavirus Disease 2019 (COVID19), has affected many facets of the practice of medicine. It has resulted in an alarming amount of hospitalizations. Since January 21, 2020, a total of 27.8 million cases have been diagnosed, in addition to 488,000 deaths in the United States alone [1]. Due to the massive spread of the pandemic, personal protective equipment (PPE) has become a part of the daily routine for healthcare workers. A majority of standard PPE worn today include the gown, gloves, N95 mask, and face shield or some form of eye protection. It has been shown by numerous studies to decrease the rate of new infections by as much as $5 \%$ over a relatively short period of time, particu- larly among healthcare workers [2]. Strict adherence to wearing PPE has been adopted in most hospitals and is also important in the procedural setting as well.

Initially, outpatient procedures were being deferred in order to minimize transmission. However, emergent surgeries and procedures would continue, and as COVID-19 became more predominant in medicine, elective procedures would also return a few months later. One of the procedures that has been closely followed is colonoscopies. Delayed diagnosis in cancer screening during the pandemic was a concern, but some studies have shown that there is no effect on cancer detection rates over a 10-month period [3]. Endoscopic procedures are considered aerosol-generating procedures which means there is a risk of transmission of viruses 
due to aerosolization when the scope is inserted and removed. The risk of aerosolization during lower GI procedures has not been well studied. Soon after the COVID-19 pandemic, the American Gastroenterological Association (AGA) issued recommendations for GI endoscopy personnel. For all GI procedures, the AGA recommends the use of N95 (or N99, or PAPR) and recommends against the use of surgical masks only, regardless of COVID-19 status [4]. The decision to extend the recommendation to lower gastrointestinal procedures is based on evidence of possible aerosolization during colonoscopy, especially during the insertion and removal of instruments through the biopsy channel and the uncertain risks associated with evidence of the presence of the viral RNA in fecal channels.

Our study looks to observe if there has been a negative effect on both the amount of screening colonoscopies and the quality measures commonly reported such as the cecal intubation rate (CIR), cecal intubation time (CIT), scope withdrawal time (SWT), and adenoma detection rate (ADR) during the pandemic period with the adoption of standard COVID-19 precautions. We hypothesized that the pandemic caused a significant decrease in the number of screening colonoscopies and other outpatient endoscopies. We also hypothesized that since the major change in protocols for a screening colonoscopy involved the use of PPE, it could possibly account for any possible changes in the quality indicators.

\section{Methods}

We conducted a retrospective chart review to analyze the effects of the COVID-19 pandemic on GI endoscopy procedures. The comparison was made during the first peak around the time when the AGA issued recommendations for GI endoscopy personnel. We compared the number of procedures performed, type of procedures, and CIR, CIT, SWT, and ADR between mid-May to mid-June (05/16$06 / 14)$ of 2019 to the same time period in $2020(05 / 18$ $06 / 16)$. The comparison was done for outpatient, nonemergent procedures conducted at 3 endoscopy suites at St. Luke's University Health Network in Bethlehem, PA, USA. The procedures themselves were performed by the same attending physicians, each of whom has at least 4-6 years of experience. The same endoscopes were used during both time periods. All inpatient and emergent colonoscopies were excluded. Data was obtained by performing a chart review on Epic electronic health records. SPSS version 26 was used to analyze the data. Missing values were not analyzed in the data. No Bonferroni correction was applied for multiple comparisons. $p$ values equal to or less than 0.05 were considered statistically significant.

\section{Results}

There were a total of 1609 patients who underwent procedures during the period of mid-May to mid-June 2019 (pre-COVID) and 1198 patients during the one-month period of mid-May to mid-June 2020 (COVID). The median age of patients undergoing endoscopy procedures was 59 in
TABLE 1: A crosstab between diagnostic/screening colonoscopies and pre-COVID/COVID time.

\begin{tabular}{lcc}
\hline Type of colonoscopy & Pre-COVID 2019 & COVID 2020 \\
& $N=1024$ & $N=637$ \\
\hline Diagnostic & $706(68.9 \%)$ & $480(75.4 \%)$ \\
Screening & $318(31.1 \%)$ & $157(24.6 \%)$ \\
\hline
\end{tabular}

$p$ value $=0.005$ (chi-squared test).

2019 and 61 in 2020. 62\% of the patients were males in 2019 as compared to $58 \%$ in 2020 . The number and type of colonoscopy were compared between the pre-COVID and COVID time periods. There was a significant decline in colonoscopies from 1024 (63.7\%) in 2019 to 637 (53.7\%) in 2020 (Table 1). Further classification was done for the colonoscopies to see the difference in the screening and diagnostic colonoscopies between the two years. The number of screening colonoscopies was almost half in 2020 compared to 2019, from 318 in pre-COVID to 157 during COVID.

Independent sample $t$-tests were done to compare CIT and SWT between the pre-COVID and COVID periods (Table 2). The mean cecal intubation time in COVID $(392 \pm 107$ seconds) was significantly higher than that in the pre-COVID group $(320 \pm 105, p=0.001)$. Similarly, scope withdrawal time in COVID (830 \pm 241 seconds) was significantly higher than that in the pre-COVID group (706 $\pm 232, p=0.001)$ whereas CIR was significantly lower in COVID $(96.60 \%)$ compared to pre-COVID time (98.20\%, $p=0.04$ ) (Table 3).

Since the data for adenoma detection rates was not normally distributed, we conducted nonparametric MannWhitney tests to compare the rates during these two periods (Table 4). It was seen that the median rate of detection during 2019 was $38.23 \%$ (12.50-66.66), and during 2020, it was $38.18 \%$ (16.66-66.00). The adenoma detection rates between these two periods were not statistically different.

\section{Discussion}

The impact of the global COVID-19 pandemic permeates every facet of medicine, including endoscopic procedures. Our study focused on seeing if implementing PPE protocols into the routine procedure of a colonoscopy could possibly affect quality indicators. Colonoscopies have been shown to play a vital role in preventing colorectal cancer (CRC). CRC screening during the pandemic overall decreased during the "lockdown period" in early 2020 due to a fear of exacerbating the spread of the virus. Modes of transmission have been studied which include aerosolization of the virus during the procedure and fecal contact spread [5]. Studies however have shown that screening colonoscopies are both safe and efficacious when performed using proper PPE and decontamination protocols for the endoscopic room after every procedure during the pandemic [6]. The likely causes of the decrease in screening colonoscopies in our study include fear on the part of the patient to participate during the pandemic and physicians deferring screening colonoscopies and only 
TABLE 2: A comparison of cecal intubation times and scope withdrawal times in screening colonoscopies between the pre-COVID-19 (2019) and COVID-19 (2020) time periods.

\begin{tabular}{lccc}
\hline & Pre-COVID 2019 & COVID 2020 & $N=648$ \\
Quality indices of colonoscopy & $N=972$ & Mean/SD (sec) & \\
& Mean/SD (sec) & $392 / 107$ & $<0.001$ \\
\hline Cecal intubation time (seconds) & $320 / 105$ & $830 / 241$ & $<0.001$ \\
Scope withdrawal time (seconds) & $706 / 232$ & & \\
\hline
\end{tabular}

*Independent sample $t$-test.

TABLE 3: A comparison of cecal intubation rates in screening colonoscopies between the pre-COVID and COVID time periods.

\begin{tabular}{lccc}
\hline Cecal intubation rates & Pre-COVID 2019 & COVID 2020 & $p$ value* \\
\hline Colonoscopies with successful intubation (N/percent) & $972 / 98.20 \%$ & $648 / 96.60 \%$ & 0.004 \\
\hline${ }^{*}$ Pearson's chi-squared test. &
\end{tabular}

TABLE 4: A comparison of adenoma detection rates between the preCOVID-19 (2019) and COVID-19 (2020) time periods.

\begin{tabular}{lcc}
\hline $\begin{array}{l}\text { Pre-COVID 2019 } \\
N=23\end{array}$ & $\begin{array}{c}\text { COVID 2020 } \\
N=22\end{array}$ & $p$ value* \\
Median (min-max) & Median (min-max) & \\
\hline $38.23 \%(12.50-66.66)$ & $38.18 \%(16.66-60.00)$ & 0.8 \\
\hline${ }^{*}$ Mann-Whitney test. &
\end{tabular}

electing for urgent/diagnostic procedures to minimize the risk of exposure.

Our study demonstrated that CIT and SWT were increased while CIR was decreased between 2019 and 2020 possibly due to PPE introduction. Before the pandemic, many factors have been shown to affect cecal intubation rates and cecal intubation times. CIR is an important quality measure that gastroenterologists are evaluated on. One study showed that the age of patients $>60$, constipation, poor preparation, and two-person colonoscopies were all independent risk factors for increased cecal intubation rates [7]. The efficiency of the procedures may be affected due to the standard precautions taken to ensure low transmission of the virus. COVID-19 precautions include thorough cleaning of the room, donning of PPE (which includes the N95 mask, gown, gloves, and face shield) for all staff during the procedures, and repeating this during turnover for every colonoscopy. This time likely translates to increased procedure time and possible decreased efficiency. This may be an independent risk factor that has not been accounted for in previous studies.

There have been few studies on this subject, but one similar study showed no difference in overall procedure time (including the cecal intubation rate) between the pre- and post-COVID-19 colonoscopy standards [8]. This was the opposite of our findings; however, this study had a lower power $(N=256)$ compared to our study which may have skewed their results. Our study demonstrated that CIR was decreased from 2019 to $2020(p=0.04)$. It is difficult to say if the implementation of PPE had a direct impact on CIR as this variable is also dependent on other factors including patient anatomy. Overall, PPE may have a negative impact on CIR and CIT. This decreased efficiency however may not have any clinical significance, and PPE continues to be necessary during the pandemic to maintain the safety of the practitioners and patients.

Another major aspect of our study focused on the comparison between $\mathrm{ADR}$ in colonoscopies prior to the implementation of PPE and in those performed after the pandemic began. ADR is distinguished from polyp detection rates $(\mathrm{PDR})$ in that the former is a subset of the latter. Some studies have attempted to provide a conversion factor from PDR to ADR [9]. ADR has been observed to be a valuable marker for cancer-related mortality. One study that reviewed over 300,000 colonoscopies found that ADR was inversely related to the risk of developing interval advanced-stage and fatal colorectal cancer [10]. In another prospective cohort study, increased ADR was associated with a decrease in cancer-related mortality [11]. We theorized that the additional PPE used during the procedure might obscure a practitioner's ability to see additional adenomas and thus affect ADR. Surprisingly, there was no difference between both groups in our results. Teh et al., as discussed prior, conducted a similar study which showed no difference in ADR between the pre- and post-COVID-19 precaution colonoscopies. This is reassuring as we see that PPE may not interfere with the clear benefits of colonoscopies.

SWT was also prolonged during colonoscopies done during the pandemic with PPE. SWT is an important measure of the efficacy of a colonoscopy. It acts as a "second pass" to detect lesions in the colon not visible on entry. Interestingly, longer SWT is associated with an increased polyp detection rate, particularly when the time is $>6$ minutes [12]. One study showed that a SWT of 10 minutes was shown to have a higher detection of overall polyps but no difference in detection of adenomatous polyps [13]. Longer SWT allows for practitioners to be more diligent in the visualization of the entire colon to the end of the procedure. In our study, SWT preCOVID was approximately 11 minutes, which was closer to the literature values, while during the COVID time period, SWT was around approximately 13 minutes. It may not have 
any clinical significance, though this may affect the amount of time a patient is under anesthesia which may lead to associated complications. Our study also found that colonoscopies performed during COVID-19 had prolonged SWT without improving ADR.

Our study did have some limitations. We acknowledge that there are confounding factors including variability between endoscopists in both expertise and experience. However, the same endoscopists performed all colonoscopies that were used between the two time periods to limit variations. The increase in CIT and SWT may or may not have clinical relevance that was observed in this study. Increased CIT and SWT may translate into more time under anesthesia and could increase complications, though our study did not focus on this. CIR and SWT are also heavily dependent on other factors as discussed prior, and PPE may or may not be directly implicated in a direct change in values. PPE may be an independent risk factor resulting in prolonged CIT and SWT while decreasing CIR without increasing ADR. Prospective studies will need to be performed to assess this, especially if COVID-19 becomes endemic and precautions continue to be required in the future.

COVID-19 has profoundly permeated every element of medicine over the past year. Colonoscopies are one of the procedures affected by the pandemic. It is vital in the prevention of CRC, and the use of PPE minimizes transmission during the procedure. Our retrospective study conveyed that there was an increased CIR and SWT. However, adenoma detection rates were similar, indicating that the use of PPE does not affect a colonoscopy's efficacy. This did not support our original hypothesis that there may be a negative impact on all quality indicators. With the advent of COVID vaccines, these precautions may change in the near future. We were encouraged to see that PPE does not interfere with the clinical benefits of colonoscopies including ADR.

\section{Conclusion}

Our study showed that quality indices for screening colonoscopies like the cecal intubation rate, cecal intubation time, and scope withdrawal time were negatively impacted during the initial COVID time period compared to pre-COVID time. The study also displayed that though there was a significant decline in both the screening and diagnostic colonoscopies during the pandemic, adenoma detection rates were comparable. Thus, the efficiency of the procedures was affected by the use of PPE but it did not affect the colonoscopy's clinical benefit.

\section{Abbreviations}

$\begin{array}{ll}\text { ADR: } & \text { Adenoma detection rate } \\ \text { CIR: } & \text { Cecal intubation rate } \\ \text { CIT: } & \text { Cecal intubation time } \\ \text { COVID-19: } & \text { Coronavirus Disease } 2019 \\ \text { CRC: } & \text { Colorectal cancer } \\ \text { PPE: } & \text { Personal protective equipment } \\ \text { SWT: } & \text { Scope withdrawal time. }\end{array}$

\section{Data Availability}

Data, analytic methods, and study materials will not be made available to other researchers.

\section{Conflicts of Interest}

There are no conflicts of interest for all authors listed.

\section{Authors' Contributions}

The contributions of the authors involved in this study are as follows: Subin Chirayath: author, editor, and data collection; Janak Bahirwani: author, editor, and data collection; Parampreet Kaur: editor and data analysis; Noel Martins: editor and data collection; and Ronak Modi: editor and principal investigator.

\section{References}

[1] CDC COVID data tracker, "Centers for Disease Control and Prevention,” 2020, http://covid.cdc.gov/covid-data-tracker/.

[2] J. L. Seidelman, S. S. Lewis, S. D. Advani et al., "Universal masking is an effective strategy to flatten the severe acute respiratory coronavirus virus 2 (SARS-CoV-2) healthcare worker epidemiologic curve," Infection Control and Hospital Epidemiology, vol. 41, no. 12, pp. 1466-1467, 2020.

[3] A. G. Dinmohamed, M. Cellamare, O. Visser et al., "The impact of the temporary suspension of national cancer screening programmes due to the COVID-19 epidemic on the diagnosis of breast and colorectal cancer in the Netherlands," Journal of Hematology \& Oncology, vol. 13, no. 1, p. 147, 2020.

[4] S. Sultan, J. K. Lim, O. Altayar et al., "AGA rapid recommendations for gastrointestinal procedures during the COVID-19 pandemic," Gastroenterology, vol. 159, no. 2, pp. 739-758.e4, 2020.

[5] R. Soetikno, A. Y. B. Teoh, T. Kaltenbach et al., "Considerations in performing endoscopy during the COVID-19 pandemic," Gastrointestinal Endoscopy, vol. 92, no. 1, pp. 176$183,2020$.

[6] V. D’Ovidio, C. Lucidi, G. Bruno, D. Lisi, L. Miglioresi, and M. E. Bazuro, "Impact of COVID-19 pandemic on colorectal cancer screening program," Clinical Colorectal Cancer, vol. 20, no. 1, pp. e5-e11, 2021.

[7] C. M. Hsu, W. P. Lin, M. Y. Su, C. T. Chiu, Y. P. Ho, and P. C. Chen, "Factors that influence cecal intubation rate during colonoscopy in deeply sedated patients," Journal of Gastroenterology and Hepatology, vol. 27, no. 1, pp. 76-80, 2012.

[8] K. K. J. Teh, S. W. Tay, K. Chen et al., "Impact of enhanced personal protective equipment on colonoscopy performance during the COVID-19 pandemic," Endoscopy international open, vol. 8, no. 6, pp. E809-E814, 2020.

[9] K. Kashiwagi, N. Inoue, T. Yoshida et al., "Polyp detection rate in transverse and sigmoid colon significantly increases with longer withdrawal time during screening colonoscopy," PLoS One, vol. 12, no. 3, article e0174155, 2017.

[10] D. L. Francis, D. T. Rodriguez-Correa, A. Buchner, G. C. Harewood, and M. Wallace, "Application of a conversion factor to estimate the adenoma detection rate from the polyp detection rate," Gastrointestinal Endoscopy, vol. 73, no. 3, pp. 493-497, 2011. 
[11] D. A. Corley, C. D. Jensen, A. R. Marks et al., "Adenoma detection rate and risk of colorectal cancer and death," The New England Journal of Medicine, vol. 370, no. 14, pp. 1298-1306, 2014.

[12] M. F. Kaminski, P. Wieszczy, M. Rupinski et al., "Increased rate of adenoma detection associates with reduced risk of colorectal cancer and death," Gastroenterology, vol. 153, no. 1, pp. 98-105, 2017.

[13] A. Ray and T. Hassan, "A study of the association between scope withdrawal time, adenoma detection rate, and polyp detection rate in a real-world setting," American Journal of Gastroenterology, vol. 107, pp. S231-S232, 2012. 